\title{
ESO 603-G21: A strange polar-ring galaxy*
}

\author{
V. P. Reshetnikov ${ }^{1,3}$, M. Faúndez-Abans ${ }^{2}$, and M. de Oliveira-Abans ${ }^{2}$ \\ 1 Astronomical Institute of St.Petersburg State University, 198504 St. Petersburg, Russia \\ 2 MCT/Laboratório Nacional de Astrofísica, Caixa Postal 21, CEP:37.504-364, Itajubá, MG, Brazil \\ e-mail: mf aundez@lna.br, mabans@lna.br \\ 3 Isaac Newton Institute of Chile, St. Petersburg Branch, Russia
}

Received 16 August 2001 / Accepted 26 November 2001

\begin{abstract}
We present the results of $B, V, R$ surface photometry of ESO 603-G21 - a galaxy with a possible polar ring. The morphological and photometric features of this galaxy are discussed. The central round object of the galaxy is rather red and presents a nearly exponential surface brightness distribution. This central structure is surrounded by a blue warped ring or disk. The totality of the observed characteristics (optical and NIR colors, strong color gradients, $\mathrm{HI}$ and $\mathrm{H}_{2}$ content, FIR luminosity and star-formation rate, rotation-curve shape, global mass-to-luminosity ratio, the agreement with the Tully-Fisher relation, etc.) shows that ESO 603-G21 is similar to late-type spiral galaxies. We suppose that morphological peculiarities and the possible existence of two large-scale kinematically-decoupled subsystems in ESO 603-G21 can be explained as being a result of dissipative merging of two spiral galaxies or as a consequence of a companion accretion onto a pre-existing spiral host.
\end{abstract}

Key words. galaxies: individual: ESO 603-G21 - galaxies: kinematics and dynamics - galaxies: photometry galaxies: formation - galaxies: structure

\section{Introduction}

The past several years have been very rich in observational studies of galaxy formation and evolution. One of the most interesting conclusions of these studies is the continuous assembly of galaxies (see Ellis 2001 for a recent review). Among the best local examples of delayed galaxy formation are the so-called multi-spin galaxies - objects with more than one axis of rotation (Rubin 1994). Polar-ring galaxies (PRG) are probably the best known instance of multi-spin objects (see Whitmore et al. 1990, hereafter PRC, for definition and catalog of such objects). PRG probably represent products of merger or external accretion phenomena (PRC, Reshetnikov \& Sotnikova 1997).

In this article we present the results of photometric observations of ESO 603-G21 - a good PRG candidate according to Whitmore et al. (1990) (see Fig. 3f in Whitmore et al. 1990 and contour maps in our Fig. 2). This galaxy resembles an early-type galaxy with a well-developed bulge and an extended warped and edge-on disk/ring. A dust

Send offprint requests to: V. P. Reshetnikov, e-mail: resh@astro.spbu.ru

* Based on observations made at the Observatório do Pico dos Dias (OPD), operated by the MCT/Laboratório Nacional de Astrofísica, Brazil. lane can be seen at the intersection of the bulge and the disk/ring.

The spectroscopic data for this object indicate a complex scenario. The rotation curves for ESO 603-G21 show that the gas and stars in the disk/ring revolve around the minor axis (PRC, Arnaboldi et al. 1994). At PA $=24^{\circ}$ (minor axis), the spectra show no motion of the gas perpendicularly to the disk/ring. In contrast, the absorption line rotation curve indicates the existence of stellar motion along this axis (Arnaboldi et al. 1994). There stellar kinematics possibly indicate that the underlying stellar body is triaxial (Arnaboldi et al. 1994; Arnaboldi et al. 1995).

\section{Observations and reductions}

The observations were performed with the $1.6-\mathrm{m}$ telescope at the OPD on August 2, 2000, equipped with direct imaging camera \#1 (details in http://www.lna.br/instrum/camara/camara.html) and CCD \#106 $(1024 \times 1024$ square pixels, $24 \mu \mathrm{m}$ each), with $\mathrm{RON}=4.1 \mathrm{e}^{-}$and gain $=5.0 \mathrm{e}^{-} / \mathrm{ADU}$.

The data were acquired with standard Johnson $B$, $V$ and Cousins $R, I$ filters. The details of the observations are given in Table 1. Photometric calibration was accomplished using standard stars from Landolt (1992). We have used the mean extinction coefficients for the OPD: $0 . \cdot 34$, 
Table 1. Observation log for August 2, 2000.

\begin{tabular}{ccccc}
\hline Bandpass & Airmass & $\begin{array}{c}\text { Exp. } \\
(\mathrm{s})\end{array}$ & $\begin{array}{c}\text { Seeing } \\
\left({ }^{\prime \prime}\right)\end{array}$ & $\begin{array}{c}\text { Sky } \\
\text { mag. }\end{array}$ \\
\hline$R$ & 1.147 & 900 & 1.0 & 20.4 \\
$R$ & 1.109 & 900 & 1.0 & 20.4 \\
$R$ & 1.078 & 900 & 1.0 & 20.4 \\
& & & & \\
$B$ & 1.052 & 900 & 1.8 & 21.5 \\
$B$ & 1.033 & 900 & 1.7 & 21.5 \\
$B$ & 1.018 & 900 & 1.8 & 21.5 \\
& & & & \\
$V$ & 1.008 & 900 & 1.7 & 20.7 \\
$V$ & 1.002 & 900 & 1.7 & 20.7 \\
$I$ & 1.001 & 900 & 1.7 & Clouds \\
\hline
\end{tabular}

0.19 and 0.14 in the $B, V$ and $R$ passbands, respectively. The $I$-band image was obtained under non-photometric conditions and so we have used it to determine the position of the galaxy nucleus only. Reduction of the raw CCD data has been made in the standard manner using the ESO-MIDAS ${ }^{1}$ package.

\section{Results}

\subsection{Integrated photometry}

The total magnitude $\left(B_{\mathrm{T}}=15.0 \pm 0.1\right)$ found by us from the multiaperture photometry is somewhat brighter than that of $\mathrm{NED}^{2}$ (15.3) and of PRC (15.5). Our $R$ magnitude $14.1 \pm 0.1$ is in agreement with the $\mathrm{PRC}$ value $m_{R}=14.07$.

Some results of color measurements are presented in Arnaboldi et al. (1994). According to those authors, the $B-R$ color index in the galaxy center is +2 , while the outer regions are rather bluer, with $B-R \approx 1$. Our values (see Figs. 3 and 4 ) are in good agreement with those results.

\subsection{Optical morphology}

In order to enhance possible internal structures of this galaxy, some tests have been carried out following the experiments performed by Faúndez-Abans and de OliveiraAbans (1998) and employing some IRAF tasks. For illustrative purposes, Fig. 1a displays the residual image of the subtraction of a $60 \times 60$-pixel median-filtered kernel from the original $R$ frame. An inner elongated bright stellar component, around another yet smaller round offcenter component have been enhanced. The dark dust lane

\footnotetext{
${ }^{1}$ MIDAS is developed and maintained by the European Southern Observatory.

2 NASA/IPAC extragalactic database.
}

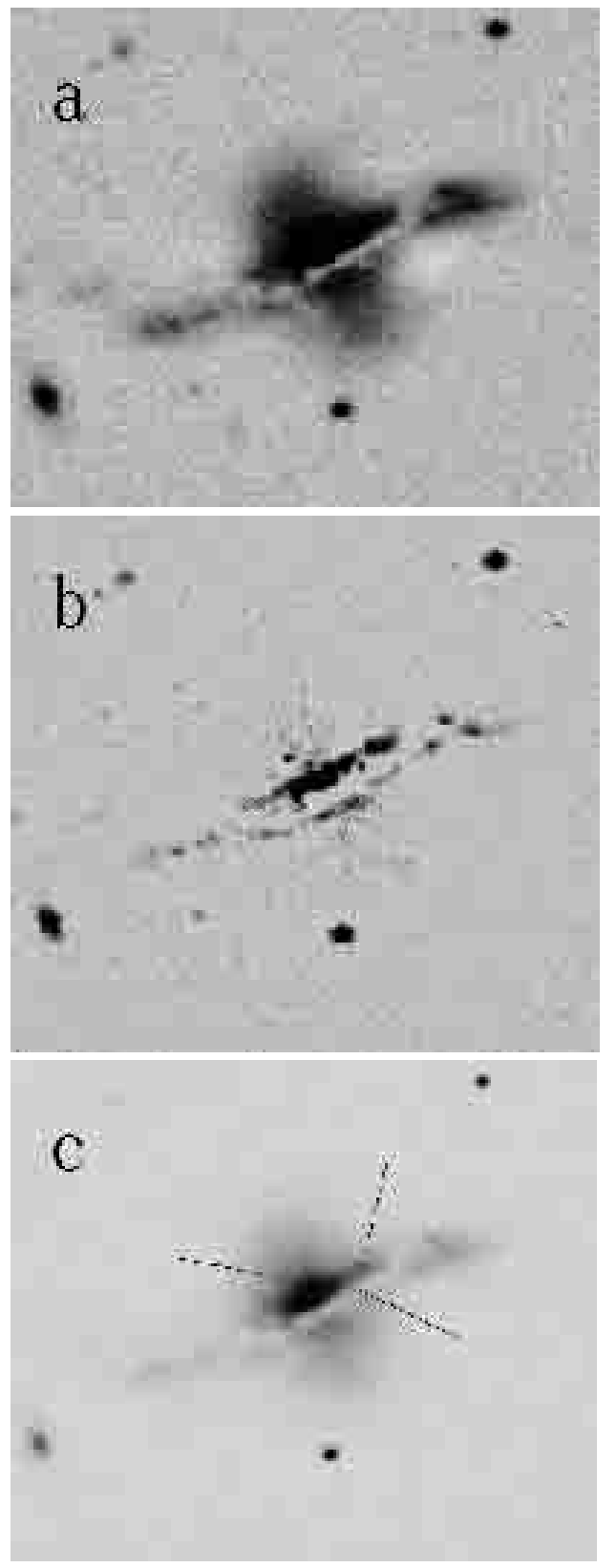

Fig. 1. a) Inverse gray scale residual image of the subtraction of the $60 \times 60$-pixel median-filtered $R$ frame from the original $R$ image. North is to the top and east is on the left. The image size is $224^{\prime \prime} \times 200^{\prime \prime}$. b) The same as a) for a $10 \times 10$-pixel kernel. c) Enhancement by transform processing and high-pass filtering of the $R$ image. The mark-lines are on purpose faint and poor so as not to cover the image.

(ring?) probably immersed in the warped material external to the disk/ring, and some faint clumps to the east have also been enhanced.

The use of a median $30 \times 30$-pixel kernel filter has enhanced the fuzzy material aligned with the apparent major axis of the bulge. Interestingly enough, after the application of a $10 \times 10$-pixel kernel median filter, this fuzzy material turned out to be filaments (see Fig. 1b). This result is in agreement with Arnaboldi et al. (1995). 
The "five to ten" filaments so-found seem to lie perpendicular to an almost edge-on exponential disk. This is indeed an interesting PR dusty galaxy.

Other features have also been revealed through a highpass filtering transform processing of the original $R$ frame: clumps (satellites?) in the bulge and within the polar ring, a faint and underlying smooth component (in the central part of the bulge), and an off-center component inside the central structure. The most prominent clumps have been indicated in Fig. 1c by thin lines. Careful visual inspection of the image display (SAO's ds9) shows that the filaments tails point towards the West, superimposed on faint smooth regions that we call lobes.

\subsection{Global photometric structure}

Contour maps of ESO 603-G21 are presented in Fig. 2. The main features of the galaxy are clearly visible: a main body with approximately round isophotes and an almost edge-on warped structure (polar ring?) crossing the central object and strongly distorting the surface brightness distribution. The galaxy is surrounded by a faint halo whose major axis is aligned with the major axis of the possible ring.

Figures 3a,c display the surface brightness profiles of ESO 603-G21 along the major and minor axes. In the major axis profile the possible disk/ring is seen as two symmetrical "bumps" at $r \approx \pm 20^{\prime \prime}$. At the SW part of the profile along the minor axis at $r \approx-2^{\prime \prime}$, a depression due to absorption in the ring/disk projected here onto the central part of the galaxy is seen.

Excluding the regions of the "bumps" $\left(|r|=10^{\prime \prime}-25^{\prime \prime}\right)$, the surface brightness distribution along the major axis may be approximated by that of an exponential disk (see also Arnaboldi et al. 1995). In the $R$ passband the disk characteristics are: $\mu_{0}^{0}(R)=19.55$ (corrected for galactic absorption) and $h=1.22 \mathrm{kpc}$. The disk of the galaxy is thus relatively bright and compact. The minor axis profile in the $R$ filter is also approximated by an exponential one with $h=3^{\prime \prime} 5=0.73 \mathrm{kpc}$ (Fig. 3 ). The total magnitude of the central exponential object is $R=14.9$ (assuming an apparent axis ratio $b / a=1.0)$. Therefore, the ratio of luminosities of the central round object (bulge?) to the disk/ring is $\sim 1$ in the $R$ passband.

Figures $3 \mathrm{~b}, \mathrm{~d}$ display the behavior of the observed color indices along the major and minor axes. Both profiles show very strong color gradients: the central parts of the galaxy are red $(B-V \approx+0.8-1.0, B-R \approx+1.5-2.0)$, while the outer ones are blue $(B-V \approx+0.2-0.5, B-R \approx+0.5-$ 1.0). The galaxy disk/ring is very blue: $B-V \approx+0.2-0.3$ and $B-R \approx+0.5$ at $r \approx \pm 20^{\prime \prime}$ along the major axis (where the two "bumps" are visible). In Fig. 3d, the region of the disk/ring projection exibits a local color minimum, thus supporting our conclusion about the blueness of the ring.

Figure 4 gives the $3 \mathrm{D}$ distribution of the observed color index $B-R$ within the central region of the galaxy.
In this figure, the disk/ring is the notably blue path (narrow "valley") crossing the central region. A prominent color gradient is evident in the figure.

In order to study the galaxy structure in the near-infrared (NIR) spectral region, we have extracted the $J, H$ and $K$ images of ESO 603-G21 from the second incremental data release of the Two Micron All Sky Survey (Skrutskie et al. 1997; see http://www.ipac.caltech.edu/2mass). The NIR colors of the galaxy (Table 2) is usual for spiral galaxies (see Fig. 9 in Iodice et al. 2001). We found that to a first approximation the galaxy structure can be described as a thick double exponential disk with strong color gradients along the major and minor axes. In Table 3 we present the scalelength ratios in different color bands, both along the major and minor axes. The large observed ratios are typical for dusty late-type spiral galaxies (e.g. de Grijs 1998).

Table 2 summarizes the main characteristics of ESO 603-G21, both found in this work and collected from the literature. The last column provides the corresponding references, where the absence of a reference indicates that the given value has been determined in this work.

\subsection{Dust and internal absorption}

The mass of warm $\left(T_{\mathrm{d}} \approx 35 \mathrm{~K}\right)$ dust found from the $100 \mu \mathrm{m}$ IRAS flux is $1.5 \times 10^{6} M_{\odot}$ (Table 2). Assuming that only $\sim 10-20 \%$ of the dust mass in disk galaxies is warm enough to radiate in the IRAS bands (Devereux \& Young 1990), we can estimate the total dust mass in the galaxy as $\sim 10^{7} M_{\odot}$.

What is the total internal absorption in ESO 603-G21? The standard empirical description of the extinction as a function of galactic inclination is

$A(i)=C \log (a / b)$

where the extinction parameter $C$ depends on the morphological type, total luminosity, and on the maximum rotaton velocity of a galaxy (e.g. de Vaucouleurs et al. 1991; Tully et al. 1998). For the purposes of this discussion, we replace the axial ratio $a / b$ with $1 / \sec i$ and take the extinction parameter in the $B$ passband from Tully et al. (1998):

$C_{B}=1.57+2.75\left[\log \left(2 V_{\max }\right)-2.5\right]$

Adopting $V_{\max }=126 \mathrm{kms}^{-1}$ (see item 3.5) and $i=$ $80^{\circ} \pm 5^{\circ}$, we obtain $A_{B}=0.99_{-0.22}^{+0.39}$. Therefore, the total extinction-corrected $B$-band absolute magnitude of ESO $603-G 21$ is $M_{B}^{0}(0)=-19.3_{-0.2}^{+0.4}$ or $L_{B}^{0}(0)=8.2 \times$ $10^{9} L_{\odot, B}$. On the other hand, the Tully-Fisher relation predicts that the extinction-corrected luminosity of a galaxy with $V_{\max }=126 \mathrm{~km} \mathrm{~s}^{-1}$ is also $M_{B}^{0}(0)=-19.3$ (Tully et al. 1998). Thus, one can conclude that $A_{B}=1{ }^{\mathrm{m}} 0$ is a reasonable estimate of the internal absorption in the galaxy and that ESO 603-G21 probably satisfies the TullyFisher relation for normal spirals. 



Fig. 2. Contour maps of ESO 603-G21 in the $B, V$ and $R$ passbands. The faintest contours in the $B, V$, and $R$ bands are 25.2 , 25.3, and 25.4 mag $\operatorname{arcsec}^{-2}$, respectively. The isophotes are separated by 0.75 mag. The large tickmarks are $20^{\prime \prime}$ apart. North is to the top and east is on the left.
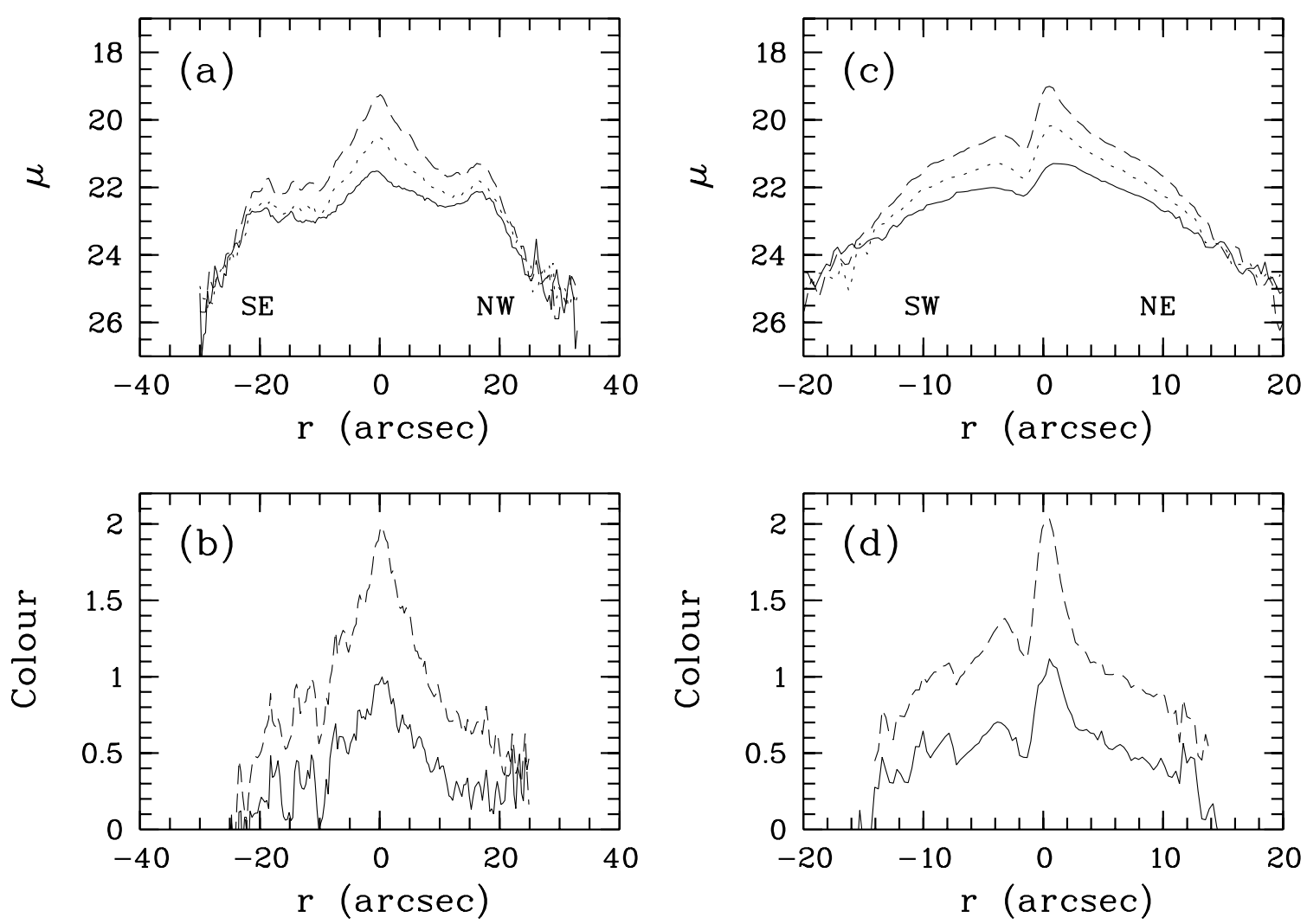

Fig. 3. Photometric profiles for ESO 603-G21: a), b) along the major axis $\left(\mathrm{PA}=114^{\circ}\right)$; c), d) along the minor axis $\left(\mathrm{PA}=24^{\circ}\right)$. Solid lines in a) and c) represent the distributions in the $B$ passband, dotted lines in $V$, and dashed ones in the $R$. Solid lines in $\mathbf{b}$ ) and $\mathbf{d}$ ) show the distribution of the $B-V$ color, dashed in the $B-R$ color.

The relatively high degree of symmetry of this object and its high inclination angle make it suitable for a study of the dust lane. In order to estimate the extinction law in the dust lane, we compare the surface brightness of regions which are equidistant from the nucleus on either side along the minor axis (see e.g. Knapen et al. 1991). Locating the exact center of the galaxy is very important for the asymmetry study. We adopt the center position as determined from the $I$-band image of ESO 603-G21. In Fig. 5 we display the selective asymmetry (which is the difference between the unobscured NE part of the minor axis profile and the SW part in one passband versus the same difference in another passband) at $r=3^{\prime \prime}-5^{\prime \prime}$ from the galaxy center (dust obscuration is probably present in the center). The mean extinction relations for the dust lane are:

$$
\begin{aligned}
& A_{B}=(1.17 \pm 0.13) A_{V} \\
& A_{R}=(0.48 \pm 0.18) A_{V} \\
& A_{R}=(0.42 \pm 0.17) A_{B}
\end{aligned}
$$


Table 2. General properties of ESO 603-G21.

\begin{tabular}{|c|c|c|}
\hline Parameter & Value & Ref. \\
\hline Morphological type & Sbc & NED \\
\hline Heliocentric systemic velocity & $3150 \mathrm{~km} \mathrm{~s}^{-1}$ & PRC \\
\hline \multirow[t]{2}{*}{ Distance } & $42.9 \mathrm{Mpc}$ & \\
\hline & $\left(1^{\prime \prime}=208 \mathrm{pc}\right)$ & \\
\hline Redshift & 0.01042 & NED \\
\hline $\mathrm{PA}$ & $114^{\mathrm{o}}$ & \\
\hline Major axis, $D_{25}\left(\mu_{B}=25\right)$ & $55^{\prime \prime}(11.4 \mathrm{kpc})$ & \\
\hline Axial ratio, $b / a\left(\mu_{B}=25\right)$ & 0.7 & \\
\hline Inclination, $i$ & $80^{\circ}:$ & \\
\hline \multicolumn{3}{|l|}{ Total apparent } \\
\hline \multicolumn{3}{|l|}{ magnitudes and colors: } \\
\hline$B_{\mathrm{T}}$ & $15.0 \pm 0.1$ & \\
\hline$(B-V)_{\mathrm{T}}$ & $+0.30 \pm 0.05$ & \\
\hline$(V-R)_{\mathrm{T}}$ & $+0.60 \pm 0.05$ & \\
\hline$(J-H)_{2 \mathrm{MASS}}$ & +0.86 & {$[1]$} \\
\hline$(H-K)_{2 \mathrm{MASS}}$ & +0.33 & {$[1]$} \\
\hline Galactic absorption ( $B$-band) & 0.14 & {$[2]$} \\
\hline Internal absorption ( $B$-band) & 1.0 & \\
\hline Absolute magnitude, $M_{B}^{0}(0)$ & -19.3 & \\
\hline \multicolumn{3}{|l|}{ Exponential disk: } \\
\hline \multicolumn{3}{|l|}{ major axis: } \\
\hline$\mu_{0}^{0}(R)$ & 19.55 & \\
\hline$h$ & $5.9(1.22 \mathrm{kpc})$ & \\
\hline \multicolumn{3}{|l|}{ minor axis: } \\
\hline$h$ & $3.5(0.73 \mathrm{kpc})$ & \\
\hline \multicolumn{3}{|l|}{ Exponential central object: } \\
\hline$R_{\mathrm{T}}$ & 14.9 & \\
\hline$L_{R}^{\text {central object }} / L_{R}^{\text {disk } / \text { ring }}$ & $\sim 1$ & \\
\hline$M(\mathrm{HI})$ & $6.2 \times 10^{9} M_{\odot}$ & {$[3,4]$} \\
\hline$M\left(\mathrm{H}_{2}\right)$ & $1.1 \times 10^{9} M_{\odot}$ & {$[5]$} \\
\hline$M(\mathrm{HI}) / L_{B}^{0}(0)$ & $0.76 M_{\odot} / L_{\odot, B}$ & \\
\hline$M\left(\mathrm{H}_{2}\right) / M(\mathrm{HI})$ & 0.18 & \\
\hline HI linewidth, $W_{20}$ & $286 \mathrm{~km} \mathrm{~s}^{-1}$ & {$[4]$} \\
\hline HI linewidth, $W_{50}$ & $251 \mathrm{kms}^{-1}$ & {$[4]$} \\
\hline Far-infrared luminosity, $L_{\mathrm{FIR}}$ & $4.85 \times 10^{9} L_{\odot}$ & $\mathrm{NED},[3]$ \\
\hline Far-infrared color, $f_{60} / f_{100}$ & 0.50 & NED \\
\hline Mass of dust, $M_{\mathrm{d}}$ & $1.5 \times 10^{6} M_{\odot}$ & {$[6]$} \\
\hline $\mathrm{SFR}_{\mathrm{FIR}}$ & $2.5 M_{\odot} / \mathrm{yr}$ & {$[7]$} \\
\hline $\operatorname{SFE}\left(=L_{\mathrm{FIR}} / M\left(\mathrm{H}_{2}\right)\right)$ & $4.4 L_{\odot} / M_{\odot}$ & \\
\hline
\end{tabular}

[1] - Skrutskie et al. (1997), [2] - Schlegel et al. (1998), [3] - Richter et al. (1994), [4] - van Driel et al. (2000), [5] - Galletta et al. (1997), [6] - Young et al. (1989), [7] - Hunter et al. (1986).
Table 3. Exponential scalelength ratios.

\begin{tabular}{|c|c|c|}
\hline Gradient & Major axis & Minor axis \\
\hline$h_{R} / h_{K}$ & $1.9 \pm 0.3$ & $1.8 \pm 0.2$ \\
$h_{J} / h_{K}$ & $1.3 \pm 0.2$ & $1.7 \pm 0.2$ \\
$h_{H} / h_{K}$ & $1.4 \pm 0.3$ & $1.4 \pm 0.3$ \\
\hline
\end{tabular}

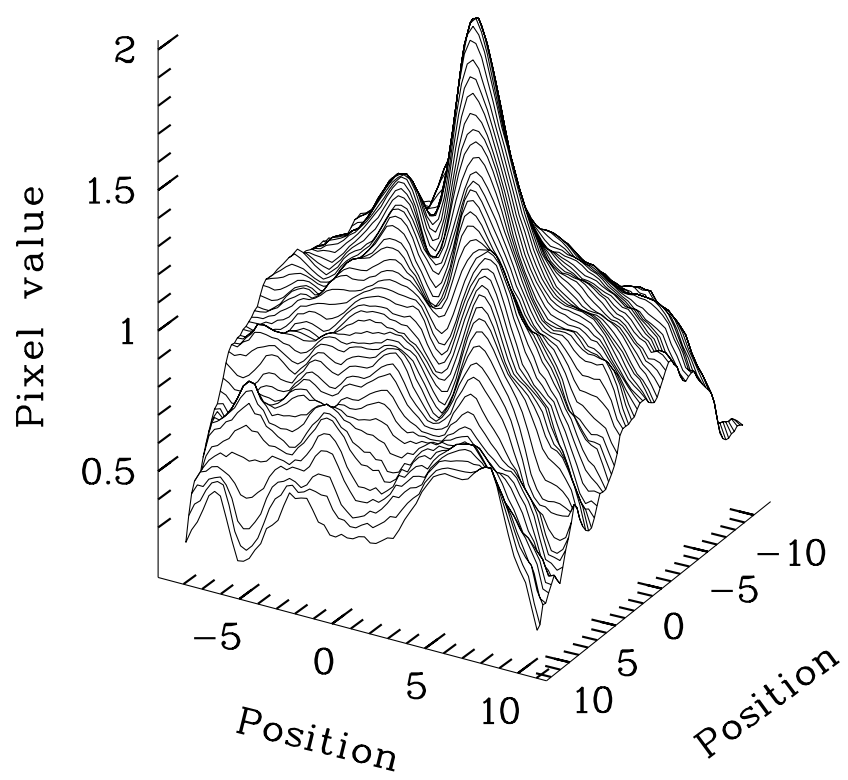

Fig. 4. A 3-dimensional distribution of the $B-R$ color index within the central $\left( \pm 10^{\prime \prime}\right)$ part of ESO $603-G 21$. The orientation is such that the line of sight coincides with the major axis of the galaxy.

The slopes of the selective asymmetry relations indicate that galactic extinction law (given as solid straight lines in Fig. 5) is valid, at least as a first approximation, for the dust in ESO 603-G21.

\subsection{Rotation curve}

The emission-line rotation curve for ESO 603-G21 along the apparent major axis $\left(\mathrm{PA}=114^{\circ}\right)$ has been published in PRC. In Fig. 6 we show the observed rotation curve of the galaxy and our fit by an exponential disk with $h=$ $1.22 \mathrm{kpc}$ (Table 2) and intrinsic axial ratio $=0.1$. It is evident that the exponential disk approximation gives a good description of the observed rotation curve within $20^{\prime \prime}$ from the nucleus.

To obtain the global maximum rotation velocity, we have used the following usual definition:

$V_{\max }=W_{50} / 2(1+z)^{-1}(\sin i)^{-1}$,

where $W_{50}$ is the HI line width at $50 \%$ of the peak, $z$ is the galaxy redshift, and $i$ is the inclination angle (see Table 2). We have obtained $V_{\max }=126 \mathrm{~km} \mathrm{~s}^{-1}$ and a total mass 

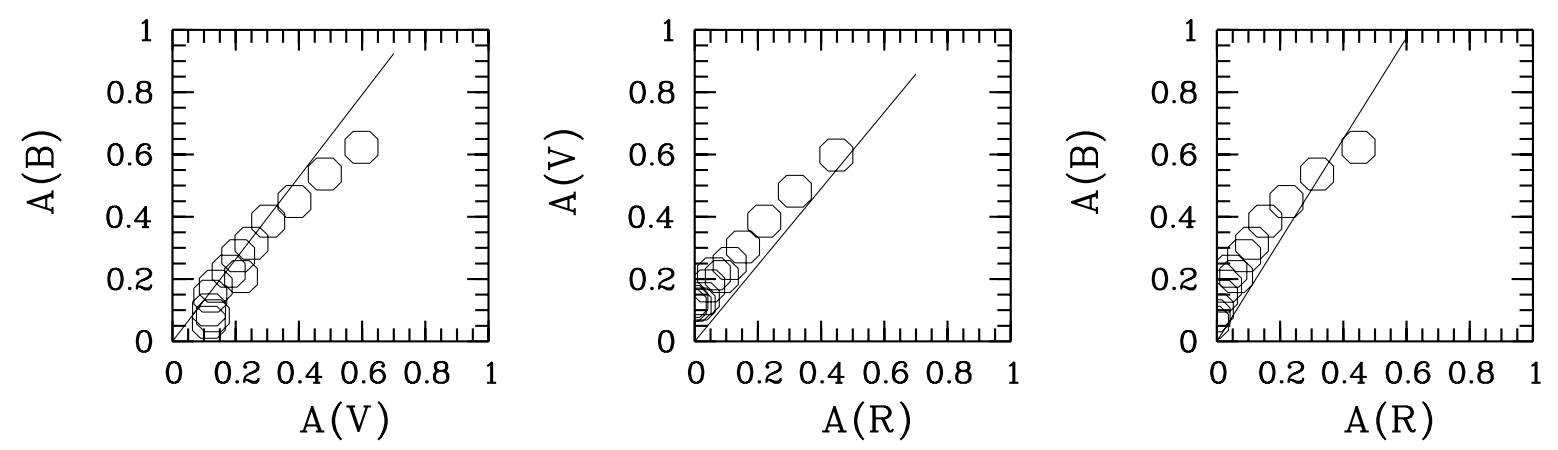

Fig. 5. Selective asymmetry for ESO 603-G21 at $3^{\prime \prime}-5^{\prime \prime}$ from the nucleus (circles). The solid line represents the galactic standard extinction law.

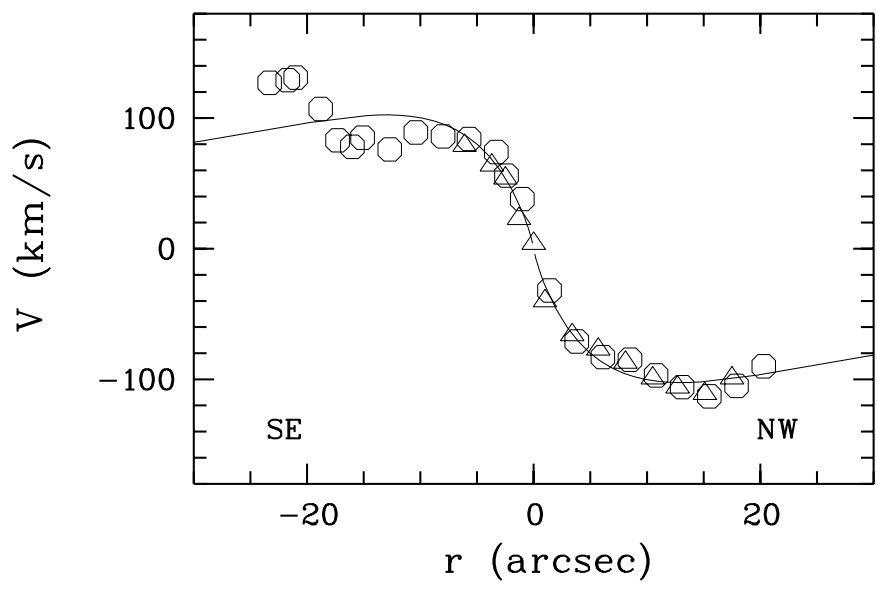

Fig. 6. Emission-line rotation curve along the major axis of ESO 603-G21 according to PRC. The circles are from $\mathrm{H} \alpha$ measurements, the triangles are from $[\mathrm{NII}]$ measurements. The solid line shows the rotation curve of an exponential disk with scalelength of $1.22 \mathrm{kpc}$.

within the optical radius $\left(R_{25}=5.7 \mathrm{kpc}\right)$ of $2.1 \times 10^{10} M_{\odot}$ (assuming a spherical mass distribution). Thus, the massto-luminosity ratio is $M / L_{B}^{0}(0)=2.6 M_{\odot} / L_{\odot, B}$, a value that is usual for disk galaxies.

\section{Discussion and conclusions}

The global kinematical structure of ESO 603-G21 - stellar rotation along two orthogonal position angles (Arnaboldi et al. 1994) - suggests that the object is a polar-ring galaxy. The host galaxy is probably an early-type galaxy with an exponential-like surface brightness distribution. The central galaxy is surrounded by a warped star-forming ring or disk. In general, ESO 603-G21 looks similar to other classic PRG (e.g. NGC 4650A).

There are, nonetheless, several facts complicating such an interpretation. First, the central round component shows very low surface brightness which may indicate that the central object is not an early-type galaxy like in most classic PRG (PRC). Second, in the near-infrared region
( $K$ passband) most of the stellar light comes from a bright nearly-exponential disk. Third, the central round object, clearly visible in the optical images (Fig. 2), is quite faint in the $K$ passband (Arnaboldi et al. 1995). The totality of the observed characteristics (optical and NIR colors, color gradients, $\mathrm{HI}$ and $\mathrm{H}_{2}$ content, FIR luminosity and star-formation rate, rotation-curve shape - Fig. $6-$, the agreement with the Tully-Fisher relation, etc.) suggests that ESO 603-G21 could be an unusual late-type spiral galaxy with a kinematically-decoupled extended "bulge". Therefore, it may be similar in some respects to NGC 4672 and NGC 4698, which are early-type disk galaxies with geometric and kinematical orthogonal decoupling between the bulge and disk (Bertola et al. 1999; Sarzi et al. 2000), or to NGC 2748, which is a late-type spiral galaxy with possible ongoing accretion of a dwarf companion onto the central region of the galaxy (Hagen-Thorn et al. 1996). The bulge-like feature of ESO 603-G21 can be a "true" polar ring that is formed during almost perpendicular accretion of an early-type companion onto central region of a pre-existing disk galaxy.

Another interesting interpretation of the observed ESO 603-G21 peculiarities is that the galaxy may be the result of a dissipative merger event (this scenario was proposed recently by Iodice et al. 2001 to explain the NGC 4650A puzzles). According to Bekki (1997, 1998), dissipative galaxy merging with a near polar orbit orientation can transform two late-type spirals into one PRG. In this scenario, a spiral galaxy falling from the polar axis of the target galaxy triggers the outwardly propogating density wave in the gaseous disk of the victim galaxy. Then, gaseous dissipation and star formation transform the victim disk into polar ring or disk. The central object is the intruding galaxy that has been turned into an early-type-like galaxy during the merging.

Figure 7 depicts the various internal substructures of ESO 603-G21 as revealed in this work (see item 3.2). Such complex, non-settled, fine structure of the galaxy supports our supposition about relatively late formation of the "bulge" due to external accretion or a merger.

Interestingly enough, the companion of ESO 603-G21 is ESO 603-G20, an edge-on disk-galaxy without any 


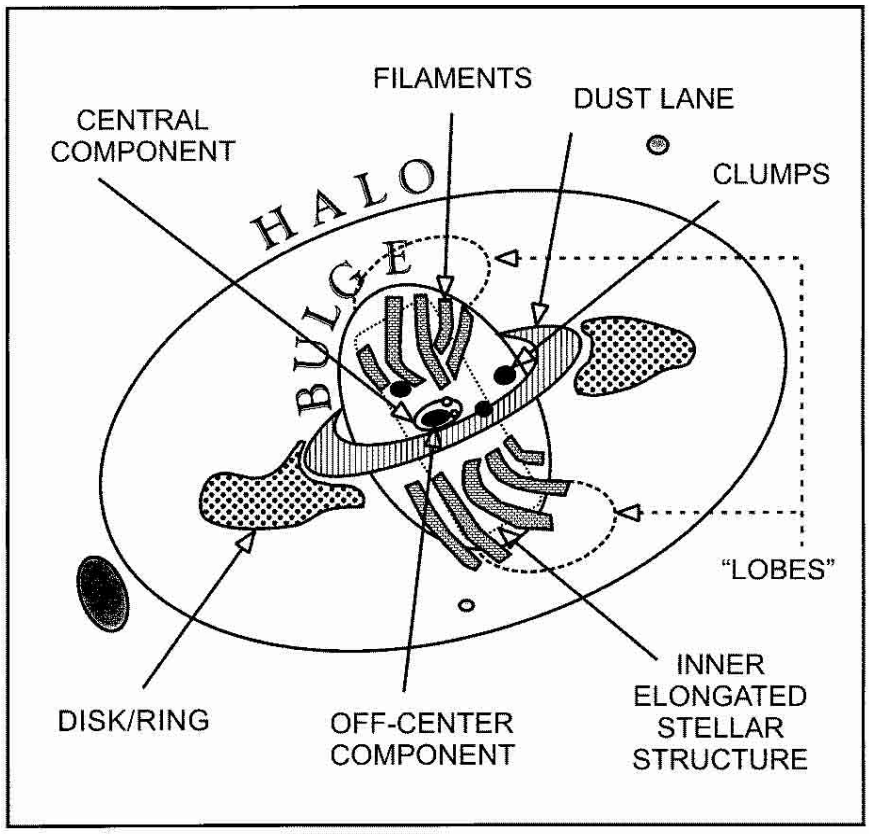

Fig. 7. Sketch of ESO 603-G21 as seen in careful visual inspection of the images in the various filters. North is up and East is on the left.

"explicit" evidence of interaction. The relative velocity between both objects is $65 \mathrm{kms}^{-1}$ (see NED and references therein); this suggests that both objects may form a bound system! There is, nonetheless, a third faint object between them, which seems to bear a very faint and narrow bridge to ESO 603-G21. The coordinates of the centroid (J2000) of this object are $\alpha=22^{\mathrm{h}} 51^{\mathrm{m}} 10.4^{\mathrm{s}}$ and $\delta=-20^{\circ} 14^{\prime} 59.5^{\prime \prime}$ within $1^{\prime \prime}$ of error, as calculated from the Digitized Sky Survey (DSS) image (see Fig. 8). Therefore, we have denoted this object Anon J225110.4-201459.5. This is probably a low-surface brightness galaxy. On the basis of the DSS image we have found that the $B$-band total magnitude of the galaxy is $B_{\mathrm{T}}=18.0 \pm 0.5$.

It is essential to note that the disks of ESO 603-G21 (Fig. 1) and ESO 603-G20 (Fig. 8) are strongly warped. This feature, as well as the probable bridge, may be an indication of ongoing interaction in the ESO 603-G21ESO 603-G20-Anon J225110.4-201459.5 triple system (e.g. Reshetnikov \& Combes 1999). So ESO 603-G21 is not an isolated object, but a member of a group of galaxies (like, for instance, NGC 4650A). Such dense spatial environment supports the idea that ESO 603-G21 may represent the result of a merging event. To test this scenario, detailed numerical simulations are needed.

Acknowledgements. We would like to thank the referee, Dr. G. Galletta, for useful comments and suggestions. V.R. acknowledges partial support from the "Integration" programme (A0145) and the DAAD (Germany). M.F.-A. and M. de O.-A. thank the partial support of the Fundação de Amparo à Pesquisa do Estado de Minas Gerais (FAPEMIG, grant

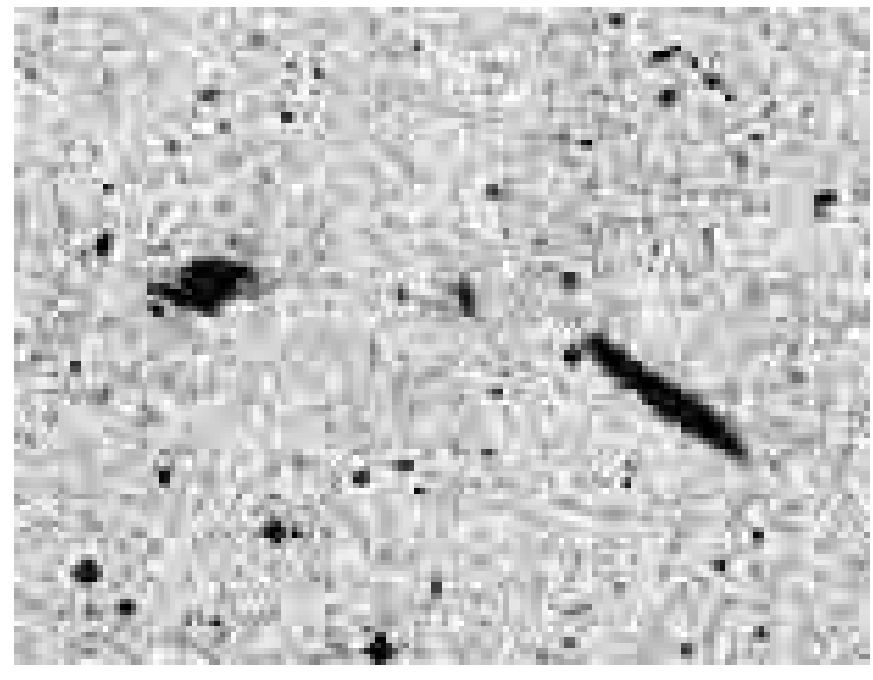

Fig. 8. In spite of the DSS resolution, a high-pass filtering has slightly enhanced the probable bridge between ESO 603-G21 (on the left) and Anon J225110.4-201459.5 at the center (see text). ESO 603-G20 is on the right. North is to the top, east on the left.

CEX 1864/95) and the Ministério da Ciência e Tecnologia (MCT, Brazil). This research has made use of the NASA/IPAC Extragalactic Database (NED) which is operated by the Jet Propulsion Laboratory, Caltech, under contract with the National Aeronautics and Space Administration. The Digitized Sky Survey was produced at the Space Telescope Science Institute (ST ScI) under US Government grant NAG W-2166. Also, this publication makes use of data products from the Two Micron All Sky Survey, which is a joint project of the University of Massachusetts and the Infrared Processing and Analysis Center/California Institute of Technology, funded by the National Aeronautics and Space Administration and the National Science Foundation.

\section{References}

Arnaboldi, M., Capaccioli, M., \& Combes, F. 1994, in The First Stromlo Symposium: The Physics of Active Galaxies, ed. G. V. Bicknell, M. A. Dopita, \& P. J. Quinn, ASP Conf. Ser., 54, 437

Arnaboldi, M., Freeman, K. C., Sackett, P. D., et al. 1995, Planet. Space Sci., 43, 1377

Bekki, K. 1997, ApJ, 490, L37

Bekki, K. 1998, ApJ, 499, 635

Bertola, F., Corsini, E. M., Vega Beltran, J. C., et al. 1999, ApJ, 519, L17

de Grijs, R. 1998, MNRAS, 299, 595

de Vaucouleurs, G., de Vaucouleurs, A., Corwin, H. G., et al. 1991, Third Reference Catalogue of Bright Galaxies (Springer-Verlag)

Devereux, N., \& Young, J. S. 1990, ApJ, 359, 42

Ellis, R. 2001 [astro-ph/0102056]

Faúndez-Abans, M., \& de Oliveira-Abans, M. 1998, A\&AS, 128,289 
Galletta, G., Sage, L. J., \& Sparke, L. S. 1997, MNRAS, 284, Sarzi, M., Corsini, E. M., Pizzella, A., et al. 2000, A\&A, 360, 773 439

Hagen-Thorn, V. A., Reshetnikov, V. P., \& Yakovleva, V. A. Schlegel, D. J., Finkbeiner, D. P., \& Davis, M. 1998, ApJ, 500, 1996, Astron. Zh., 73, 36 525

Hunter, D. A., Gillett, F. C., Gallagher, J. S., et al. 1986, ApJ, 303, 171

Iodice, E., Arnaboldi, M., De Lucia, G., et al. 2001, AJ, accepted [astro-ph/0110249]

Knapen, J. H., Hes, R., Beckman, J. E., \& Peletier, R. F. 1991, A\&A, 241, 42

Landolt, A. U. 1992, AJ, 104, 340

Reshetnikov, V., \& Sotnikova, N. 1997, A\&A, 325, 933

Reshetnikov, V., \& Combes, F. 1999, A\&AS, 138, 101

krutskie, M. F., Schneider, S. E., Stiening, R., et al. 1997, in The Impact of Large Scale Near-IR Sky Surveys, ed. F. Garzon, et al. (Dordrecht, Kluwer), 25

Tully, R. B., Pierce, M. J., Huang, J.-S., et al. 1998, AJ, 115, 2264

van Driel, W., Arnaboldi, M., Combes, F., \& Sparke, L. S. 2000, A\&AS, 141, 385

Whitmore, B. C., Lucas, R. A., McElroy, D. B., et al. 1990, AJ, 100, 1489 (PRC)

Richter, O.-G., Sacket, P. D., \& Sparke, L. S. 1994, AJ, 107, Young, J. S., Xie, S., Kenney, J. P. D., \& Rice, W. L. 1989, 99 ApJS, 70, 699

Rubin, V. C. 1994, AJ, 108, 456 